\title{
Complicaciones postoperatorias graves tras esofagectomía para carcinoma esofágico: análisis de factores de riesgo
}

\author{
FERNANDO ARMÉSTAR ${ }^{a}$, EDUARD MESALLES ${ }^{a}$, ALBERT FONT $^{b}$, ANTONIO ARELLANO $^{c}$, JOSEP ROCA $^{d}$, \\ JORDI KLAMBURG ${ }^{a}$ Y JAIME FERNÁNDEZ-LLAMAZARES \\ aServicio de Medicina Intensiva. Hospital Universitario Germans Trias i Pujol. \\ Universidad Autónoma de Barcelona. Badalona. Barcelona. España. \\ bServicio de Oncología. Hospital Universitario Germans Trias i Pujol. \\ Universidad Autónoma de Barcelona. Badalona. Barcelona. España. \\ 'Servicio de Radioterapia. Hospital Universitario Germans Trias i Pujol. \\ Universidad Autónoma de Barcelona. Badalona. Barcelona. España. \\ ¿Servicio de Epidemiología. Hospital Universitario Germans Trias i Pujol. \\ Universidad Autónoma de Barcelona. Badalona. Barcelona. España. \\ eServicio de Cirugía. Hospital Universitario Germans Trias i Pujol. \\ Universidad Autónoma de Barcelona. Badalona. Barcelona. España.
}

Introducción. Estudio realizado para identificar los factores de riesgo de morbilidad y mortalidad postoperatorias en pacientes sometidos a esofagectomía por cáncer de esófago.

Diseño y pacientes. Estudio retrospectivo llevado a cabo en un centro hospitalario español. La población estudiada fueron 159 pacientes con cáncer de esófago localmente avanzado, a los que se realizó esofagectomía, entre enero de 1985 y diciembre de 2004.

Resultados. El 54\% de enfermos presentaron complicaciones graves, de las que las más frecuentes fueron las de tipo infeccioso. La mortalidad de la serie fue del $12,5 \%$. El fallo multiorgánico secundario a sepsis fue la causa de muerte más frecuente. Las complicaciones graves postoperatorias mostraron una asociación significativa con: alcoholismo ( $p<0,04)$, la cirrosis hepática $(p<0,03)$, la localización del tumor en el tercio medio del esófago $(p<0,04)$, y el índice de APACHE II > 10 (p<0,003). La mortalidad se asoció significativamente con la presencia de enfermedad pulmonar crónica $(p=0,03)$ y con un índice de APACHE II > 10 ( $p=0,02)$.

Correspondencia: Dr. F. Arméstar

Hospital Universitario Germans Trias i Pujol. Universidad Autónoma de Barcelona.

Ctra. del Canyet, s/n. 08916 Badalona. Barcelona. España.

Correo electrónico: 33800far@comb.es

Manuscrito aceptado el 1-12-2008
Conclusiones. EI APACHE II > 10 puede utilizarse como factor pronóstico tanto de mortalidad como de complicaciones graves. La enfermedad pulmonar crónica es un factor que se asocia a mayor mortalidad postoperatoria. El abuso de alcohol, la cirrosis hepática y la localización del tumor esofágico son factores que se asocian a complicaciones graves postoperatorias.

PALABRAS CLAVE: Esofaguectomía. Postoperatorio. Complicaciones.

\section{SERIOUS POSTOPERATIVE COMPLICATIONS AFTER ESOPHAGECTOMY FOR ESOPHAGEAL CARCINOMA: ANALYSIS OF RISK FACTORS}

Introduction. To identify risk factors for postoperative morbidity and mortality in patients undergoing esophagectomy for esophageal cancer.

Design and patients. The population comprised 159 patients with locally advanced esophageal cancer, undergoing esophagectomy between January 1985 and December 2004.

Results. Infections were the main cause of both complications and postoperative mortality. The $\mathbf{5 4 \%}$ of our patients presented serious complications. The mortality of the series was $12.5 \%$. Multiorganic failure secondary to sepsis was the more frequent cause of death. The postoperative complications showed a significant association with: alcoholism ( $p<0.04)$, hepatic cirrhosis $(p<0.03)$, the location of the tumor in middle third 
of the esophagus ( $p<0.04)$, and the APACHE II score greater of 10 ( $p<0.003)$. Mortality was associated significantly with the presence of chronic pulmonary disease $(p=0,03)$ and with an APACHE II score superior to $10(p=0,02)$.

Conclusions. The APACHE II score superior to 10 can be used so much as prognostic factor of mortality like of serious complications. Chronic obstructive pulmonary disease is a risk factor for postoperative mortality. Alcoholism, hepatic cirrhosis and the location of the tumor, are factors associated to postoperative serious complications

KEY WORDS: Esophagectomy. Postoperative. Complications.

\section{INTRODUCCIÓN}

La supervivencia de los pacientes que se someten a cirugía por carcinoma esofágico localmente avanzado ha mejorado durante los últimos años. Los avances en la técnica quirúrgica y el manejo perioperatorio son, en gran parte, causa de esta mejoría ${ }^{1}$. A pesar de ello, estos pacientes continúan presentando una alta incidencia de complicaciones postoperatorias ${ }^{2}$.

Son varios los factores de riesgo identificados como predictores de una mala evolución postoperatoria; sin embargo, hay discrepancias a la hora de su identificación, pues al analizar las series publicadas no todos los autores encuentran los mismos factores de riesgo y algunos presentan datos que contradicen los hallazgos de otros ${ }^{3-5}$.

Asimismo, se han descrito técnicas, como la analgesia epidural, cuyo uso se ha relacionado con una mejor evolución postoperatoria ${ }^{6}$.

La mejora en la selección preoperatoria de los pacientes mediante sistemas de valoración del estado general y de las enfermedades concomitantes ${ }^{7,8}$, el manejo postoperatorio en una unidad de cuidados intensivos ${ }^{9}$ y que la intervención se realice en un centro con un alto número de esofagectomías ${ }^{10}$ son factores que han incidido de forma positiva en la disminución de la tasa de complicaciones de la cirugía esofágica.

El objetivo de este estudio es identificar los factores asociados con aparición de complicaciones graves y mortalidad postoperatoria, lo que nos permitiría desarrollar estrategias que optimicen el manejo perioperatorio en este tipo de pacientes. Como objetivo secundario se describen las complicaciones y la mortalidad de la serie de pacientes.

\section{PACIENTES Y MÉTODO}

Se estudió retrospectivamente una serie de 159 enfermos que habían sido sometidos consecutivamente a esofagectomía por cáncer de esófago localmente avanzado.

El estudio se realizó en el Hospital Universitario Germans Trias i Pujol de Badalona. Un mismo equipo de cirujanos operó a los pacientes entre enero de 1985 y diciembre de 2004. A partir de 1991 se introdujeron la quimioterapia y la radioterapia preoperato- rias para los pacientes con carcinoma escamoso en estadios II y III. Por este motivo incluimos dentro de las variables dos periodos evolutivos. El primero desde 1985 hasta 1990 y el segundo de 1991 a 2004. La información se obtuvo mediante revisión de las historias clínicas.

\section{Variables analizadas}

Parámetros preoperatorios: las variables cualitativas fueron sexo, alcoholismo, tabaquismo, enfermedades crónicas concomitantes, tipo histológico del tumor, localización del tumor, tratamiento preoperatorio, periodo evolutivo y estadificación TNM. Las cuantitativas: edad, hemograma, pruebas de estado nutricional (proteínas, albúmina, colesterol, índice de masa corporal), pruebas de coagulación, función renal y hepática.

Parámetros operatorios: las variables cualitativas fueron tiempo de cirugía, radicalidad de la resección (cirugía radical: R0, cirugía parcial: R1). Las cuantitativas: número de concentrados de hematíes transfundidos.

Parámetros postoperatorios durante las primeras 24 horas: las variables cuantitativas fueron APACHE II, hemograma, pruebas de coagulación, pruebas de estado nutricional y función renal y hepática las primeras 24 horas postoperatorias.

Consideramos alcoholismo la ingesta $>35$ unidades ( 1 unidad equivale a $8 \mathrm{~g}$ de alcohol al $100 \%$ ) por semana para los varones y más de 21 unidades por semana para las mujeres ${ }^{11}$. La enfermedad pulmonar obstructiva crónica fue diagnosticada por criterios clínicos y pruebas funcionales respiratorias $^{12}$. Consideramos que la resección no fue completa cuando la información anatomopatológica de la pieza quirúrgica detectó afección transmural del tumor (R1). La media del tiempo total de cirugía incluyó la preparación anestésica y la colocación del paciente.

\section{Complicaciones postoperatorias graves}

Consideramos complicaciones postoperatorias graves las que ponen en peligro la vida de los pacientes. Fueron las siguientes: infecciosas (sepsis grave, shock séptico), fallo multiorgánico (FMO), pulmonares no infecciosas (síndrome de distrés respiratorio, atelectasia masiva, broncoaspiración, neumotórax, parálisis de cuerdas vocales), hemorrágicas, cardiovasculares (shock cardiogénico, edema agudo de pulmón, angina inestable, fibrilación ventricular, tromboembolia pulmonar), digestivas (insuficiencia hepática, hemorragia digestiva) y renales (insuficiencia renal que requiere diálisis).

Las definiciones utilizadas para sepsis grave, shock séptico y FMO fueron las establecidas según la conferencia de consenso del American College of Chest/ Society of Critical Care Medicine en $1991^{16}$ y la Conferencia Internacional de definiciones de sepsis de la SCCM/ESCIM/ACCP/ATS/SIS del $2001^{17}$. 


\section{Mortalidad postoperatoria}

Definimos mortalidad postoperatoria como las muertes ocurridas los primeros 30 días postoperatorios. Las causas de muerte analizadas se clasificaron como: fallo multiorgánico (FMO), shock hemorrágico, insuficiencia respiratoria, shock séptico y shock cardiogénico. Entre los fallecimientos por shock séptico incluimos a los pacientes que murieron por shock séptico resistente al tratamiento que no llegaron a sufrir FMO.

\section{Valoración de la enfermedad}

Se basó en la realización de una fibroendoscopia alta con biopsia de la tumoración esofágica, una tomografía computarizada (TC) toracoabdominal, y en los últimos años se ha agregado la ecografía endoscópica. Se practicó a todos los pacientes pruebas de función pulmonar, radiografía de tórax, electrocardiograma, cuantificación de su estado general, parámetros analíticos (que incluyeron hemograma, pruebas de estado nutricional, función renal y pruebas de función hepática). El manejo postoperatorio se realizó en la unidad de medicina intensiva.

\section{Técnica quirúrgica}

El abordaje quirúrgico comprendió una laparatomía en la línea media y la movilización del estómago, seguida de una toracotomía derecha a nivel del quinto espacio intercostal con resección del esófago y del estómago proximal.

Los pacientes fueron colocados en decúbito lateral izquierdo; se colapsó el pulmón derecho mediante intubación orotraqueal selectiva durante la fase de toracotomía.

La continuidad gastrointestinal se restauró usando el estómago en la mayoría de las ocasiones, excepto en los casos con gastrectomías previas, en los que se utilizó el colon. La anastomosis fue cervical o intratorácica. Se realizó linfadenectomía en bloque en dos campos.

\section{Manejo postoperatorio}

Los pacientes después de quirófano ingresaban sistemáticamente en la unidad de medicina intensiva y todos llegaban de quirófano manteniendo la ventilación mecánica.

El protocolo de actuación en cuidados intensivos incluía: $a$ ) antibioticoterapia profiláctica; $b$ ) aspiración mediante sonda nasogástrica; $c$ ) analgesia epidural o intravenosa desde el ingreso; $d$ ) extubación cuando se cumplían criterios de extubación ${ }^{15} ; e$ ) retirada de drenajes torácicos, y $f$ ) esofagograma con contraste no iónico a partir del quinto día postoperatorio.

No se realizaron transfusiones sanguíneas a menos que se evidenciara una cifra de hemoglobina $\leq 7 \mathrm{~g} /$ $\mathrm{dl}^{16} \mathrm{o}$ cuando la situación clínica del paciente lo requiriera.
Se administró nutrición parenteral durante los primeros días, y después se continuó con nutrición enteral por yeyunostomía.

Se controló el dolor con analgesia epidural o intravenosa con opiáceos.

Se administró heparina subcutánea para la profilaxis de la enfermedad tromboembólica. El último año se añadieron las medias de compresión neumática.

\section{Análisis estadístico}

Para describir la distribución de variables, utilizamos las distribuciones de frecuencia, media, mediana y error estándar de la media para variables continuas y los porcentajes para variables categóricas.

La asociación entre variables independientes y complicaciones postoperatorias o mortalidad se determinó mediante análisis univariable, usando el test de la $\chi^{2}$, el test exacto de Fisher o el test de dos muestras de Wilcoxon. Para determinar el factor pronóstico independiente de complicaciones y mortalidad, usamos modelos de regresión logística. Se calculó la odds ratio (OR) ajustada para estimar el valor pronóstico independiente. Para estimar la mediana de supervivencia de esta serie de pacientes, usamos el método de Kaplan-Meier.

Para todos los análisis y comparaciones, se usó el valor de $\mathrm{p}<0,05$ para determinar la significación estadística. Todas las estadísticas se realizaron mediante el paquete de software de SAS para Windows, versión 9.1.

\section{RESULTADOS}

La tabla 1 resume las características de la población estudiada.

\section{Complicaciones graves}

El 54\% de los pacientes (87) sufrieron un total de 136 complicaciones graves, descritas en la tabla 2 .

\section{Complicaciones infecciosas}

Fueron las más frecuentes de esta serie de pacientes; $60(37,7 \%)$ presentaron sepsis grave, shock séptico o FMO (el origen de la infección se describe en la tabla 3). El foco más frecuente fue pulmonar $(15 \%)$, seguido de las fugas mediastínicas $(9,5 \%)$.

\section{Complicaciones pulmonares no infecciosas}

Se presentaron en 47 (29,5\%) pacientes. La más frecuente fue el SDRA (34 pacientes). Otras fueron: atelectasia masiva (8), neumotórax a tensión (2), quilotórax (2) y parálisis de cuerdas vocales (1). Los pacientes con quilotórax no fueron reoperados. 
TABLA 1. Características de la población

\begin{tabular}{|c|c|}
\hline Parámetro & Población $(n=159)$ \\
\hline Edad (años) & $58 \pm 9$ \\
\hline Varones $(\%)$ & 92 \\
\hline Fumadores (\%) & 71 \\
\hline Alcoholismo (\%) & 57 \\
\hline $\operatorname{EPOC}(\%)$ & 34 \\
\hline Cirrosis hepática (\%) & 9 \\
\hline Cardiopatía $(\%)$ & 9 \\
\hline Insuficienca renal crónica $(\%)$ & 3 \\
\hline $\mathrm{DM}(\%)$ & 3 \\
\hline Índice de masa corporal & $0,36 \pm 1,58$ \\
\hline \multicolumn{2}{|l|}{ Localización del tumor } \\
\hline Tercio medio $(\%)$ & 54 \\
\hline Tercio superior $(\%)$ & 37 \\
\hline Tercio inferior $(\%)$ & 9 \\
\hline \multicolumn{2}{|l|}{ Tipo de cáncer } \\
\hline CE $(\%)$ & 81 \\
\hline $\operatorname{ADK}(\%)$ & 19 \\
\hline Tratamiento preoperatorio* $(\%)$ & 48 \\
\hline \multicolumn{2}{|l|}{ Albúmina sérica } \\
\hline Preoperatorio (g/l) & $38 \pm 4,7$ \\
\hline Postoperatorio $(\mathrm{g} / \mathrm{l})$ & $27,4 \pm 6,11$ \\
\hline \multicolumn{2}{|l|}{ Proteínas } \\
\hline Preoperatorio (g/l) & $64,5 \pm 6,0$ \\
\hline Postoperatorio (g/l) & $44,0 \pm 10,9$ \\
\hline \multicolumn{2}{|l|}{ Colesterol } \\
\hline Preoperatorio (g/l) & $182,3 \pm 45$ \\
\hline Postoperatorio (g/l) & $185,6 \pm 24$ \\
\hline \multicolumn{2}{|l|}{ Hemoglobina } \\
\hline Preoperatorio (g/dl) & $12,4 \pm 2$ \\
\hline Postoperatoria (g/dl) & $130 \pm 3$ \\
\hline \multicolumn{2}{|l|}{ Tiempo de protrombina $(\%)$} \\
\hline Preoperatorio & $92 \pm 11$ \\
\hline Postoperatorio & $67 \pm 11$ \\
\hline Tiempo de cirugía $(\mathrm{h})$ & $5,03 \pm 1$ \\
\hline Transfusión de hematíes (unidades) & $1,6 \pm 1,4$ \\
\hline $\mathrm{R} 1(\%)$ & 17 \\
\hline Leucocitos $\left(\times 10^{9} / 1\right)$ postoperatorios & $10,6 \pm 7,3$ \\
\hline Plaquetas $\left(\times 10^{9} / 1\right)$ postoperatorias & $195 \pm 49,7$ \\
\hline \multicolumn{2}{|l|}{ Creatinina } \\
\hline Preoperatoria (mg/dl) & $1,0 \pm 1,4$ \\
\hline Postoperatoria (mg/dl) & $0,7 \pm 1,6$ \\
\hline APACHE II & $12,1 \pm 3,2$ \\
\hline Ventilación mecánica (días) & $6,8 \pm 10$ \\
\hline \multicolumn{2}{|l|}{ Pacientes por periodo (n) } \\
\hline $1985-1990$ & 30 \\
\hline 1991-2004 & 129 \\
\hline
\end{tabular}

ADK: adencoarcinoma; CE: carcinoma escamoso; DM: diabetes mellitus; EPOC: enfermedad pulmonar obstructiva crónica.

*Referido sólo a pacientes con carcinoma escamoso.

Los datos expresan frecuencia (porcentaje) o media \pm desviación estándar.

\section{Complicaciones cardiovasculares}

Presentaron shock cardiogénico 5 pacientes. Otras fueron EAP (3 casos), angina inestable (2), TEP (1) y fibrilación ventricular (1).

\section{Hemorragia postoperatoria}

Desarrollaron shock hemorrágico 12 pacientes; por este motivo 5 de ellos fueron reintervenidos de forma inmediata, con éxito. De ellos, en 3 se encontró rotura esplénica, en 1 caso sangrado arterial de
TABLA 2. Complicaciones postoperatorias ( $n=159)$

\begin{tabular}{|lcc|}
\hline Complicaciones & $\mathrm{n}(\%)$ & IC del $95 \%$ \\
\hline Infecciosas & $60(37,7)$ & $25,5-49,7$ \\
Pulmonares & $24(15)$ & $1-29$ \\
Dehiscencia de & $15(9,5)$ & $0-23,5$ \\
$\quad$ sutura & & \\
Abdominal sin & $8(5)$ & $0-20$ \\
$\quad$ dehiscencia & $2(1,2)$ & \\
Catéter & $11(7)$ & $0-13$ \\
No filiado & $47(29,5)$ & $0-22$ \\
Respiratorias & $12(7,5)$ & $16,5-42$ \\
Hemorrágicas & $9(5,6)$ & $0,5-21,5$ \\
Cardiovasculares & $6(3,7)$ & $0-18,7$ \\
Digestivas & $3(1,8)$ & $0-16,8$ \\
Renales & & \\
\hline
\end{tabular}

TABLA 3. Causas de muerte

\begin{tabular}{|lcc|}
\hline Causas & $\mathrm{n}(\%)$ & IC del 95\% \\
\hline Fallo multiorgánico & $13(62)$ & $36-88$ \\
Shock hemorrágico & $3(19)$ & $0-63$ \\
Insuficiencia respiratoria & $2(9)$ & $0-29$ \\
Shock séptico & $1(5)$ & $0-47$ \\
Shock cardiogénico & $1(5)$ & $0-47$ \\
Total & $20(100)$ & \\
\hline
\end{tabular}

una rama de la aorta y en otro no se encontró punto de sangrado.

\section{Complicaciones digestivas}

Presentaron insuficiencia hepática aguda 4 pacientes y hemorragia digestiva alta, 2 .

\section{Complicaciones renales}

Tres pacientes presentaron insuficiencia renal con requerimiento de diálisis.

\section{Mortalidad}

La mortalidad postoperatoria total fue del $12,5 \%$ (20 pacientes). Las causas de la muerte se describen en la tabla 3.

La mortalidad durante la primera etapa (19851990) alcanzó un $27 \%$. Sin embargo, en la segunda etapa (1991-2004) la mortalidad fue del 9\%.

El FMO fue la causa más frecuente de muerte: 13 (62\%) pacientes. En 8 de ellos fue secundario a fugas mediastínicas; en 2 se debió a infección pulmonar, y en 1 fue secundario a isquemia intestinal. No se filió el origen en 2 pacientes.

El shock hemorrágico fue la segunda causa de muerte; 2 pacientes fallecieron por sangrado postoperatorio inmediato y 1 con hemorragia digestiva alta; la insuficiencia respiratoria por SDRA fue la causa de muerte en 2 pacientes; el shock séptico sin evolución a FMO fue causa de muerte en 1 paciente, y 1 paciente falleció en shock cardiogénico durante las primeras 24 horas del postoperatorio. 
TABLA 4. Factores asociados con complicaciones graves postoperatorias. Análisis univariable

\begin{tabular}{|c|c|c|c|}
\hline Parámetro & Complicados $(\mathrm{n}=87)$ & $\begin{array}{l}\text { No complicados } \\
\quad(\mathrm{n}=72)\end{array}$ & $\mathrm{p}$ \\
\hline Edad (años) & $58 \pm 9$ & $58 \pm 10$ & NS \\
\hline Sexo: varones/mujeres & $16,2 / 1$ & $9,4 / 1$ & NS \\
\hline Fumadores $(\%)$ & 76 & 64 & NS \\
\hline Alcoholismo (\%) & 65 & 48 & 0,03 \\
\hline EPOC $(\%)$ & 33 & 35 & NS \\
\hline Cirrosis hepática (\%) & 15 & 4 & 0,009 \\
\hline Cardiopatía (\%) & 10 & 8 & NS \\
\hline Insuficienca renal crónica (\%) & 2 & 4 & NS \\
\hline Diabetes mellitus (\%) & 4 & 1 & NS \\
\hline Índice de masa corporal (IMC) & 0,23 & 0,23 & NS \\
\hline \multicolumn{4}{|l|}{ Localización del tumor } \\
\hline Tercio medio (\%) & 45 & 27 & 0,02 \\
\hline Tercio superior $(\%)$ & 7 & 11 & NS \\
\hline Tercio inferior $(\%)$ & 48 & 61 & NS \\
\hline \multicolumn{4}{|l|}{ Tipo de cáncer } \\
\hline $\mathrm{CE}(\%)$ & 83 & 76 & NS \\
\hline $\operatorname{ADK}(\%)$ & 16 & 23 & NS \\
\hline Tratamiento preoperatorio* $(\%)$ & 52 & 42 & NS \\
\hline CE según tratamiento preoperatorio & $38(61)$ & $24(39)$ & \\
\hline $\mathrm{CE}$ con tratamiento preoperatorio, $\mathrm{n}(\%)$ & & & \\
\hline $\mathrm{CE}$ sin tratamiento preoperatorio, $\mathrm{n}(\%)$ & $34(51)$ & $32(49)$ & \\
\hline \multicolumn{4}{|l|}{ Albúmina sérica } \\
\hline Preoperatoria (g/l) & 37,2 & 39 & NS \\
\hline Postoperatoria (g/l) & 26 & 26 & NS \\
\hline \multicolumn{4}{|l|}{ Proteínas } \\
\hline Preoperatorias (g/l) & 69,7 & 64 & NS \\
\hline Postoperatorias $(\mathrm{g} / \mathrm{l})$ & 45 & 45 & NS \\
\hline \multicolumn{4}{|l|}{ Colesterol } \\
\hline Preoperatorio (g/l) & 179,6 & 185 & NS \\
\hline Postoperatorio (g/l) & 187 & 198 & NS \\
\hline \multicolumn{4}{|l|}{ Hemoglobina } \\
\hline Preoperatoria (g/dl) & 12,4 & 12,4 & NS \\
\hline Postoperatoria (g/dl) & 12,9 & 12,9 & NS \\
\hline \multicolumn{4}{|l|}{ Tiempo de protrombina $(\%)$} \\
\hline Preoperatorio & 91 & 93 & NS \\
\hline Postoperatorio & 71 & 73 & NS \\
\hline Tiempo de cirugía (h) & 5,1 & 4,95 & NS \\
\hline Transfusión de hematíes (unidades) & 1,7 & 1,44 & NS \\
\hline \multicolumn{4}{|l|}{$\mathrm{R} 1(\%)$} \\
\hline Leucocitos $\left(\times 10^{9} / 1\right)$ postoperatorios & 11,9 & 10,5 & NS \\
\hline Plaquetas $\left(\times 10^{9} / 1\right)$ postoperatorias & 185,9 & 205 & NS \\
\hline Creatinina $(\mathrm{mg} / \mathrm{dl})$ & 1,05 & 0,95 & NS \\
\hline APACHE II & 11 & 9 & 0,0012 \\
\hline Ventilación mecánica (días) & 13,6 & 1,7 & 0,001 \\
\hline \multicolumn{4}{|l|}{ Periodo } \\
\hline 1985-1990, n (\%) & $18(60)$ & $12(40)$ & NS \\
\hline $1991-2004, \mathrm{n}(\%)$ & $69(53)$ & $60(47)$ & \\
\hline
\end{tabular}

*Sólo pacientes con carcinoma escamoso.

\section{Análisis de los parámetros}

En las tablas 4 y 5 se analizan los parámetros preoperatorios, intraoperatorios y postoperatorios, comparándolos según hubiera o no complicaciones graves.

La media de edad de nuestra serie era 58 años, y la mayoría de los pacientes eran varones.

Las siguientes variables se asociaron a un postoperatorio tórpido: el alcoholismo, la cirrosis hepática y la localización en el tercio medio del esófago. Asimismo, el índice de APACHE II > 10 también fue un factor asociado a complicaciones postoperatorias.
TABLA 5. Factores asociados con complicaciones graves postoperatorias. Análisis multivariante

\begin{tabular}{|l|c|c|}
\hline Parámetro & OR (IC del 95\%) & $\mathrm{p}$ \\
\hline Alcoholismo & 5,0 & 0,04 \\
& $(1,03-24,2)$ & \\
Cirrosis hepática & 0,4 & 0,03 \\
& $(1,08-5,4)$ & \\
Tumor en tercio & 4,10 & 0,04 \\
$\quad$ medio & $(1,05-16,0)$ & \\
APACHE II $>10$ & 2,9 & 0,003 \\
& $(1,4-5,8)$ & \\
\hline
\end{tabular}


En la tablas 6 y 7 se analizan los parámetros preoperatorios, intraoperatorios y postoperatorios, según la mortalidad.

\section{Supervivencia}

La mediana de supervivencia de esta serie de enfermos fue 15 meses.

\section{DISCUSIÓN}

En esta serie de pacientes encontramos relación entre tener un hábito alcohólico importante, padecer cirrosis hepática y tener el tumor localizado en el tercio medio del esófago con aparición de complicaciones graves postoperatorias. El índice de APACHE II también se demostró buen predictor de un postoperatorio tórpido. Los factores pronósticos asociados a mortalidad postoperatoria fueron el APACHE II y la EPOC.

El abuso del alcohol es un conocido factor etiológico del carcinoma escamoso de esófago ${ }^{17}$, pero también es un importante predictor de morbilidad postoperatoria ${ }^{18}$, como demostramos en nuestro trabajo. El consumo crónico de alcohol se asocia a arritmias cardíacas, cardiomiopatías, alteraciones circulatorias, complicaciones hemorrágicas y estado de inmunodeficiencia ${ }^{19}$ que predispone a una mayor incidencia de infecciones. La inclusión de medidas

TABLA 6. Factores asociados con mortalidad postoperatoria. Análisis univariable

\begin{tabular}{|c|c|c|c|}
\hline Parámetro & Fallecidos $(\mathrm{n}=20)$ & $\operatorname{Vivos}(\mathrm{n}=139)$ & $\mathrm{p}$ \\
\hline Edad (años) & $61,8 \pm 9$ & $57,9 \pm 9$ & NS \\
\hline Varones/mujeres & $6 / 1$ & $14 / 1$ & NS \\
\hline Fumadores (\%) & 70 & 71 & NS \\
\hline Alcoholismo (\%) & 60 & 57 & NS \\
\hline $\mathrm{EPOC}(\%)$ & 65 & 29 & 0,0017 \\
\hline Cirrosis hepática (\%) & 27 & 11 & NS \\
\hline Cardiopatía (\%) & 5 & 9 & NS \\
\hline Insuficienca renal crónica (\%) & 10 & 2 & NS \\
\hline Diabetes mellitus (\%) & 10 & 3 & NS \\
\hline Índice de masa corporal & 0,22 & 0,23 & NS \\
\hline \multicolumn{4}{|l|}{ Localización del tumor } \\
\hline Tercio medio $(\%)$ & 50 & 55 & NS \\
\hline Tercio superior (\%) & 45 & 36 & NS \\
\hline Tercio inferior (\%) & 5 & 9 & NS \\
\hline \multicolumn{4}{|l|}{ Tipo de cáncer } \\
\hline CE $(\%)$ & 80 & 88 & NS \\
\hline $\operatorname{ADK}(\%)$ & 20 & 19 & \\
\hline \multicolumn{4}{|l|}{ Tratamiento preoperatorio* $(\%)$} \\
\hline CE según tratamiento preoperatorio & 20 & 41 & NS \\
\hline $\mathrm{CE}$ con tratamiento preoperatorio & $5(18)$ & $57(82)$ & NS \\
\hline $\mathrm{CE}$ sin tratamiento preoperatorio & $12(8)$ & $54(92)$ & NS \\
\hline \multicolumn{4}{|l|}{ Albúmina sérica } \\
\hline Preoperatoria (g/l) & 37,2 & 39 & NS \\
\hline Postoperatoria (g/l) & 24 & 27 & NS \\
\hline \multicolumn{4}{|l|}{ Proteínas } \\
\hline Preoperatorias (g/l) & 64,7 & 64 & NS \\
\hline Postoperatorias (g/l) & 43 & 46 & NS \\
\hline \multicolumn{4}{|l|}{ Colesterol } \\
\hline Preoperatorio (g/l) & 179,6 & 185 & NS \\
\hline Postoperatorio (g/l) & 71 & 99 & NS \\
\hline \multicolumn{4}{|l|}{ Hemoglobina } \\
\hline Preoperatoria (g/dl) & 12,4 & 12,4 & NS \\
\hline Postoperatoria (g/dl) & 13 & 13 & NS \\
\hline \multicolumn{4}{|l|}{ Tiempo de protrombina } \\
\hline Preoperatorio & 91 & 93 & NS \\
\hline Postoperatorio & 61 & 73 & NS \\
\hline Tiempo de cirugía (h) & 5,1 & 4,95 & NS \\
\hline Transfusión de hematíes (unidades) & 1,7 & 1,44 & NS \\
\hline R1 (\%) & 20 & 13 & NS \\
\hline Leucocitos $\left(\times 10^{9} / 1\right)$ postoperatorios & 10 & 11 & NS \\
\hline Plaquetas $\left(\times 10^{9} / 1\right)$ postoperatorias & 162 & 207 & NS \\
\hline Creatinina $(\mathrm{mg} / \mathrm{dl})$ & 1,1 & 0,98 & NS \\
\hline APACHE II & 13 & 10 & 0,0015 \\
\hline \multicolumn{4}{|l|}{ Pacientes por periodo, $\mathrm{n}(\%)$} \\
\hline $1985-1990$ & $8(27)$ & $22(73)$ & 0,02 \\
\hline $1991-2004$ & $12(9)$ & $117(91)$ & \\
\hline
\end{tabular}

*Sólo pacientes con carcinoma escamoso. 
TABLA 7. Factores asociados con mortalidad postoperatoria. Análisis multivariable

\begin{tabular}{|l|c|c|}
\hline Parámetro & OR (IC del 95\%) & $\mathrm{p}$ \\
\hline EPOC & 3,2 & 0,03 \\
& $(1,132-9,687)$ & \\
APACHE II $>10$ & 0,2 & 0,02 \\
& $(0,06-0,7)$ & \\
\hline
\end{tabular}

que consigan un largo periodo de abstinencia previa a la cirugía podría conseguir disminuir la morbilidad postoperatoria.

Nuestro estudio encontró una asociación entre la cirrosis hepática y complicaciones postoperatorias graves. Los trabajos de Bartels et $\mathrm{al}^{7}$ y Nagawa et al ${ }^{8}$ encontraron una asociación entre cirrosis hepática y la aparición de complicaciones pulmonares postoperatorias. Una posible explicación sería el estado de desnutrición y la tendencia a retención de líquidos en el tercer espacio que tienen estos enfermos. Aunque está claro que la cirrosis hepática avanzada es una contraindicación para la cirugía, incluso algunos enfermos con cirrosis compensada tienen un riesgo incrementado de sufrir complicaciones. Por ello el estudio de Bartels et $\mathrm{al}^{7}$ propone el uso del test respiratorio de aminopiri$\mathrm{na}^{20}$, mediante el cual se podría discriminar a los pacientes con disfunción hepática y un riesgo aumentado de morbilidad postoperatoria.

La localización del tumor en el tercio superior fue, según el estudio de Law et $\mathrm{al}^{21}$, un factor de riesgo de complicaciones pulmonares. En nuestra serie de pacientes, los tumores de localización en el tercio medio fueron los que presentaron mayor morbilidad postoperatoria. Una explicación para esta diferencia podría estar en el bajo número de pacientes con tumor esofágico de localización superior en nuestra serie.

El APACHE II sólo o combinado con otros índices es un contrastado predictor de mortalidad de enfermos graves en diferentes condiciones patológicas ${ }^{22}$. En nuestro estudio, una puntuación de APACHE II $>10$ se asoció con mayor riesgo de mortalidad y complicaciones graves postoperatorias.

Aunque algunos estudios encuentran que la duración prolongada de la ventilación mecánica es un factor que por sí mismo se asocia a mayor morbilidad postoperatoria $^{23}$, es difícil poder separar el hecho bien conocido de que la ventilación mecánica prolongada es también consecuencia de la situación de gravedad del paciente; lo que está claro es que es un indicador de gravedad. Nosotros observamos que un tiempo de ventilación mecánica superior a 3 días nos podría indicar que el paciente puede estar iniciando una complicación grave.

En cuanto a la EPOC, como en el trabajo de Steyeberg et $\mathrm{al}^{24}$, también encontramos una asociación con la mortalidad. Es conocida la asociación de esa enfermedad durante el postoperatorio con el incremento de atelectasias, neumonías, destete difícil, prolongación de la ventilación mecánica y de la estancia hospitalaria e incremento de la mortalidad. Por ello, en este tipo de pacientes se debe rea- lizar un tratamiento agresivo (broncodilatadores, fisioterapia, antibióticos si están indicados, corticoides y el cese del hábito de fumar) que permita que lleguen a las mejores condiciones para la cirugía ${ }^{25}$.

La mortalidad postoperatoria del total de la serie fue del $12,5 \%$ (20 pacientes). Al analizarla por periodos, observamos una mortalidad de $27 \%$ en el primero (1985-1990), mientras que en el segundo (19912004) fue del $9 \%$. A pesar de ello, en el análisis multivariable esta diferencia no alcanza significación, posiblemente porque fueron enfermos con características y tipo de selección diferentes. Las cifras de mortalidad después de una esofagectomía oscilan entre el 4 y el $11 \%$, 26,27 aunque hay autores que han registrado cifras de mortalidad mucho más bajas. Casi todos los estudios coinciden en que con el paso de los años la mortalidad de este tipo de pacientes ha disminuido enormemente.

Existe un gran número de publicaciones sobre las complicaciones de la cirugía esofágica, todas ellas coinciden en la alta morbilidad de este tipo de enfermos $^{8}$. Nuestro trabajo encontró que un 54\% de los pacientes tuvieron complicaciones graves, de las que las más frecuentes fueron las infecciosas (38\%), principalmente las de origen respiratorio. Un factor que puede contribuir a la alta incidencia de complicaciones infecciosas es el estado de inmunodeficiencia ocasionado tanto por la radioterapia y la quimioterapia previas a la cirugía como por el mismo procedimiento quirúrgico ${ }^{4,28}$.

Las infecciones respiratorias se presentaron en el $15 \%$ de los pacientes. La incidencia de este tipo de infecciones después de la esofagectomía varía ampliamente, del 7 al $17 \%{ }^{32}$, así como su contribución a la mortalidad, que para algunos autores es la principal causa de muerte postoperatoria ${ }^{23}$. En el periodo inicial, después de la esofagectomía se produce un mayor riesgo de aspiración debido a alteraciones en la deglución, y el riesgo es mucho mayor si hay lesión del nervio laríngeo recurrente ${ }^{30}$. Asimismo, son importantes los factores mecánicos innatos a la cirugía torácica como el dolor, la disminución de la movilidad torácica, las atelectasias, la tos ineficaz y la disminución del aclaramiento de secreciones. Son varios los factores que pueden influir en la disminución de las infecciones pulmonares. El cese del hábito tabáquico por lo menos 4 a 6 semanas antes de la cirugía, el adecuado tratamiento preoperatorio de los pacientes con EPOC, la utilización de la analgesia torácica epidural con destete temprano de la ventilación mecánica, la realización de broncoscopias con tanta frecuencia como el paciente requiera, la adecuada fisioterapia respiratoria y la posición semisentada son factores que influyen en la disminución de la neumonía tras cirugía esofágica ${ }^{31}$. En nuestro estudio no se utilizó analgesia epidural en todos los enfermos.

Las fugas anastomóticas -que fueron la segunda causa de infecciones- se presentaron en el 10\% de los pacientes. La incidencia de fugas anastomóticas (3$14 \%$ ) y la mortalidad asociada a ella varía en función 
de cada una de las series publicadas. Algunos autores coinciden con nuestra serie de enfermos en que es la principal causa de muerte postoperatoria ${ }^{29,32}$. De los procedimientos utilizados para evitar esta complicación, destaca la analgesia epidural ${ }^{6}$, a la que se atribuye un efecto vasodilatador en la microcirculación intestinal y un incremento de la oxigenación tisular de los pacientes sometidos a cirugía abdominal ${ }^{33}$. Aunque hay controversia en cuanto al manejo de las fugas mediastínicas, hay acuerdo en utilizar el tratamiento quirúrgico cuando se presente una situación de fallo multiorgánico ${ }^{34,35}$.

De las complicaciones respiratorias no infecciosas, la principal fue el SDRA, que se presentó en el $21 \%$ de los pacientes. Tandon et $\mathrm{al}^{35}$, en una serie de 168 pacientes esofagectomizados, encontraron que un $14,5 \%$ de los pacientes desarrolló un SDRA. La aparición de SDRA tiene relación con estados inflamatorios sistémicos como el shock séptico; pero además, en estos pacientes se puede producir lesión pulmonar por mecanismos de isquemia y reperfusión, secundaria a la ventilación intraoperatoria de un solo pulmón (colapso y reexpansión del pulmón) ${ }^{36}$. Asimismo, la estrategia ventilatoria es también un aspecto a destacar. Un estudio observó que al aplicar la ventilación protectora (VT: $5 \mathrm{ml} / \mathrm{kg}$ ) durante la fase de colapso de un pulmón permitía un destete de la ventilación mecánica más precoz ${ }^{37}$. Se han ensayado con resultados positivos los glucocorticoides, que administrados de forma profiláctica podrían prevenir la aparición del SDRA ${ }^{38}$. El manejo restrictivo de líquidos durante la intervención quirúrgica también podría tener repercusión en la disminución de las complicaciones pulmonares ${ }^{39}$.

La principal causa de muerte fue el fallo multiorgánico (13 pacientes) secundario a un proceso infeccioso; el foco más frecuente fue la fuga anastomótica. Resultados similares a los obtenidos por la serie de Griffin et $\mathrm{al}^{32}$.

\section{Limitaciones y críticas}

Nuestro estudio tiene la limitación de ser retrospectivo. Tiene dos épocas diferenciadas en las cuales la mortalidad es diferente. Esto limita las conclusiones, a pesar de que el equipo de cirujanos e intensivistas ha sido el mismo. El predominio de una localización de tumor sobre otra podría estar influido por la diferente procedencia de los enfermos, pues nuestro hospital es un centro de referencia.

La serie presentada tiene en general altas tasas de complicaciones y mortalidad, por ello una optimización en todas las etapas del manejo de estos pacientes, desde la selección hasta los cuidados postoperatorios, nos permitiría una reducción de nuestras tasas de morbilidad y mortalidad ${ }^{7,40}$.

En conclusión, las infecciones, predominantemente las de origen pulmonar y las secundarias a fugas mediastínicas, son la causa más frecuente de una evolución postoperatoria tórpida. El abuso del alcohol, la cirrosis hepática, la localización del tumor y el índice de APACHE II se asocian con complicaciones graves postoperatorias. El fallo multiorgánico secundario a sepis es la más frecuente causa de muerte. La EPOC y el APACHE II > 10 son factores pronósticos de mortalidad.

\section{BIBLIOGRAFÍA}

1. Ando N, Ozawa S, Kitagawa Y, Shinozawa Y, Kitajima M. Improvement in the results of surgical treatment of advanced squamous esophageal carcinoma during 15 consecutive years. Ann Surg. 2000;232:225-32.

2. González-González JJ, Sanz-Álvarez L, Marqués-Álvarez L, Navarrete-Guijosa F, Martínez-Rodríguez E. Complications of surgical resection of esophageal cancer. Cir Esp. 2006;80:349-60.

3. Eguchi R, Ide H, Nakamura T, Hayashi K, Ohta M, Okamoto $\mathrm{F}$, et al. Analysis of postoperative complications after esophagectomy for esophageal cancer in patients receiving neoadjuvant therapy. JJTCVS. 1999;47:552-8.

4. Heidecke CD, Weighardt H, Feith M, Fink U, Zimmermann F, Stein H, et al. Neoadyuvant treatment of esophageal cancer: Immunosupresion following combined radiochemotherapy. Surgery. 2002;132:495-501.

5. Siewert JR, Stein HJ, Feith M, Bruecher BL, Bartels H, Fink $\mathrm{U}$. Histologic tumor type is an independent prognostic parameter in esophageal cancer: lessons from more than 1,000 consecutive resections at a single center in the Western world. Ann Surg. 2001;234:360-7.

6. Michelet P, D'Journo X-B, Roch A, Papazian L, Ragni J, Thomas, et al. Perioperative risk factors for anastomotic leakage after esophagectomy: influence of thoracic epidural analgesia. Chest. 2005;128:3461-6.

7. Bartels H, Stein H, Siewert J. Preoperative risk analysis and postoperative mortality of oesophagectomy for respectable oesophageal cancer. Br J Surg. 1998;85:840-4.

8. Nagawa H, Kobori O, Muto T. Prediction of Pulmonary complications after trasthoracic oesophagectomy. Br J Surg. 1994; 81:860-2.

9. Dimick J, Pronovost P, Heitmiller R, Lipsett P. Intensive care unit physician staffing is associated with decreased length of stay, hospital cost, and complications after esophageal resection. Crit Care Med. 2001;29:753-8.

10. Begg C, Cramer L, Hoskins W, Brennan M. Impact of hospital volume on operative mortality for major surgery. JAMA. 1998;280:1747-51.

11. Royal College of Psychiatrists. Alcohol: our favourite drug. New report of a Special Committee of the Royal College of Psychiatrists. London: Tavistock; 1986. p. 176-8.

12. Official statement of the American Thoracic Society. Standards for the diagnosis and care of patients with chronic obstructive pulmonary disease. Am J Respir Crit Care Med. 1995;152: S77-120.

13. Members of the American College of Chest Physiicans/ Society of Critical Care Med Consensus Conference Committee: American College of Chest Physicians/society of Crit Care Med Consensus Conference: Definitions for sepsis and organ failure and guidelines for the use of innovative therapies in sepsis. Crit Care Med. 1992;20:864-74.

14. Levy MM, Fink MP, Marshall JC, Abraham E, Angus D, Cook D, et al. 2001 SCCM/ESCIM/ACCP/ATS/SIS Internacional Sepsis Definitions Conference. Crit Care Med. 2003;31:1250-6.

15. Vallverdú I, Calaf N, Subirana M, Net A, Benito S, Mancebo J. Clinical characteristics, respiratory functional parameters, and outcome of a two-hour t-piece trial in patients weaning from mechanical ventilation. Am J Respir Crit Care Med. 1998;158:1855-62.

16. Consensus Conference (National Institures of Health): Perioperative red blood cell transfusion. JAMA. 1988;260:2700-3.

17. Tsutsumi M, George J, Ishizawa K, Fukumura A, Takase S. Effect of chronic dietary ethanol in the promotion of N-nitrosomethylbenzylamine-induced esophageal carcinogenesis in rats. J Gastroenterol Hepatol. 2006;21:805-13. 
18. Tonnesen H, Kehlet H. Preoperative alcoholism and postoperative morbidity. Br J Surg. 1999;86:869-74.

19. Spies CD, Von Dossow V, Eggers V, Jetschmann G, El-Hilali R, Egert J, et al. Altered cell-mediated immunity and increased postoperative infection rate in long-term alcoholic patients. Anesthesiology. 2004;100:1088-100.

20. Gill RA, Goodman MW, Golfus GR, Onstad GR, Bubrick MP. Aminopyrina breath test predicts surgical risk for patients with liver disease. Ann Surg. 1983;198:701-4.

21. Law S, Wong KH, Kwok KF, Chu KM, Wong J. Predictive factors for postoperative pulmonary complications and mortality after esophagectomy for cancer. Ann Surg. 2004;240:791-800.

22. Mayr VD, Dünser MW, Greil V, Jochberger S, Luckner G, Ulmer $\mathrm{H}$, et al. Causes of death and determinants of outcome in critically ill patients. Am J Crit Care. 2006;15:47-53.

23. Caldwell, Murphy P, Walsh T, Hennessy T. Timing of extubation after oesophagectomy. Br J Surg. 1993;80:1537-9.

24. Steyerberg E, Neville B, Koppert L, Lemmens V, Tilanus H, Coebergh JW, et al. Surgical mortality in patients with esophageal cancer: development and validation of a simple risk score. J Clin Oncol. 2006;24:4277-84.

25. Smetana GW. Preoperative pulmonary evaluation. N Engl J Med. 1999;340:937-44.

26. Biondi A, Rausi S, Zoccali M, Vigorita V, Persiani R. Postoperative care after esophagectomy: The surgeon's view. Rays. 2005:30:299-307.

27. Bailey S, Bull D, Harpole D, Rentz J, Neumayer L, Pappas $\mathrm{T}$, et al. Outcomes after esophagectomy: a ten-year prospective cohort. Ann Thorac Surg. 2003;75:217-22

28. Hensler T, Hecker T, Heeg K, Bartels H, Barthlen W, Wagner $\mathrm{H}$, et al. Distinct mechanisms of immunosuppression as a consequence of major surgery. Infect Inmunol. 1997;65:2283-91.

29. Robertson SA, Skipworth RJ, Clarke DL, Crofts TJ, Lee A, De Beaux AC, et al. Ventilatory and intensive care requirements following oesophageal resection. Ann R Coll Surg Engl. 2006;88:354-7.
30. Hetmiller RF, Tseng E, Jones B. Prevalence of aspiration and laryngeal penetration in patients with unilateral vocal fold motion impairment. Dysphagia. 2000;15:184-7.

31. Biondi A, Rausi S, Zoccali M, Vigorita V, Persiani R. Postoperative care after esophagectomy: The surgeon's view. Rays. 2005;30:299-307

32. Griffin M, Shaw I, Dresner M. Early complications after Ivor Lewis subtotal esophagectomy with two-field lymphadenectomy: risk factors and management. J Am Coll Surg. 2002;194285.

33. Kabon B, Fleischmann E, Treschan T. Thoracic epidural anesthesia increases tissue oxygenation during major abdomina surgery. Anesth Analg. 2003;97:1812-7.

34. Sauvanet A, Baltar J, Mee L, Belghiti J. Diagnosis and conservative management of intrathoracic leakage after oesophagectomy. Br J Surg. 1998;85:1446-9.

35. Tandon S, Batchelor A, Bullock R, Gascoigne A, Griffin M, Hing $\mathrm{J}$, et al. Perioperative risk factors for acute lung injury after elective oesophagectomy. Br J Anaesth. 2001;86:633-8.

36. Jordan J, Mitchell JA, Quinlan GJ, Goldstraw P, Evans TW. The Pathogenesis of lung injury following pulmonary resection. Eur Resp J. 2000;15:790-9.

37. Michelet P, D'Journo XB, Roch A, Doddoli C, Marin V, Papazian L, et al. Protective ventilation influences systemic inflamation after esophagectomy: a randomized controlled study. Anesthesiology. 2006;105:911-9.

38. Takeda S, Ogawa R, Nakanishi K, Kim C, Miyashita M, Sasajima K, et al. The effect of preoperative high dose methylprednisolone in attenuating the metabolic response after oesophageal resection. Eur J Surg. 1997;163:511-7.

39. Kita T, Mammoto T, Kishi Y. Fluid management and postoperative respiratory disturbances in patients with transthoracic esophagectomy for carcinoma. J Clin Anesth. 2002;14:252-6.

40. Gockel I, Sultanov FS, Domeyer M, Goenner U, Junginger T. Developments in esophageal surgery for adenocarcinoma: a comparison of two decades. BMC Cancer. 2007;7:114. 